\title{
Prevascularization promotes endogenous cell-mediated angiogenesis by upregulating the expression of fibrinogen and connective tissue growth factor in tissue- engineered bone grafts
}

Pengzhen Cheng ${ }^{1 \dagger}$, Donglin $\mathrm{Li}^{1,2+}, \mathrm{Yi} \mathrm{GaO}^{1}$, Tianqing Cao ${ }^{1}$, Huijie Jiang ${ }^{1}$, Jimeng Wang ${ }^{1,3}$, Junqin $\mathrm{Li}^{1}$, Shuaishuai Zhang ${ }^{1}$, Yue Song ${ }^{1}$, Bin Liu', Chunmei Wang ${ }^{1}$, Liu Yang ${ }^{1 *}$ and Guoxian Pei ${ }^{1^{*}}$

\begin{abstract}
Background: Vascularization is one of the most important processes in tissue-engineered bone graft (TEBG)mediated regeneration of large segmental bone defects. We previously showed that prevascularization of TEBGs promoted capillary vessel formation within the defected site and accelerated new bone formation. However, the precise mechanisms and contribution of endogenous cells were not explored.

Methods: We established a large defect $(5 \mathrm{~mm})$ model in the femur of EGFP ${ }^{+}$transgenic rats and implanted a $\beta$ tricalcium phosphate ( $\beta$-TCP) scaffold seeded with exogenous EGFP- ${ }^{-}$cells; the femoral vascular bundle was inserted into the scaffold before implantation in the prevascularized TEBG group. Histopathology and scanning electron microscopy were performed and connective tissue growth factor (CTGF) and fibrin expression, exogenous cell survival, endogenous cell migration and behavior, and collagen type I and III deposition were assessed at 1 and 4 weeks post implantation.

Results: We found that the fibrinogen content can be increased at the early stage of vascular bundle transplantation, forming a fibrin reticulate structure and tubular connections between pores of $\beta$-TCP material, which provides a support for cell attachment and migration. Meanwhile, CTGF expression is increased, and more endogenous cells can be recruited and promote collagen synthesis and angiogenesis. By 4 weeks post implantation, the tubular connections transformed into von Willebrand factor-positive capillary-like structures with deposition of type III collagen, and accelerated angiogenesis of endogenous cells.
\end{abstract}

Conclusions: These findings demonstrate that prevascularization promotes the recruitment of endogenous cells and collagen deposition by upregulating fibrinogen and CTGF, directly resulting in new blood vessel formation. In addition, this molecular mechanism can be used to establish fast-acting angiogenesis materials in future clinical applications.

Keywords: Prevascularization, Angiogenesis, Tissue-engineered bone grafts, Fibrinogen, Connective tissue growth factor

\footnotetext{
* Correspondence: yangliu@fmmu.edu.cn; nfperry@163.com

${ }^{+}$Pengzhen Cheng and Donglin Li contributed equally to this work.

${ }^{1}$ Institute of Orthopedic Surgery, Xijing Hospital, Fourth Military Medical

University, Xi'an 710032, People's Republic of China

Full list of author information is available at the end of the article
}

(c) The Author(s). 2018 Open Access This article is distributed under the terms of the Creative Commons Attribution 4.0 International License (http://creativecommons.org/licenses/by/4.0/), which permits unrestricted use, distribution, and reproduction in any medium, provided you give appropriate credit to the original author(s) and the source, provide a link to the Creative Commons license, and indicate if changes were made. The Creative Commons Public Domain Dedication waiver (http://creativecommons.org/publicdomain/zero/1.0/) applies to the data made available in this article, unless otherwise stated. 


\section{Background}

Large segmental bone defects caused by severe trauma, tumor, and pathological fractures usually fail to heal naturally due to the limited self-repairing capabilities $[1,2]$. Over the last two decades, regenerative medicine and bone tissue engineering techniques have offered promising alternative approaches for the treatment of large bone defects without side effects compared with traditional therapies [3-6].

Vascularization is one of the most important processes in TEBG-mediated regeneration of large segmental bone defects. Angiogenesis is a highly regulated event involving complex, dynamic interactions between vascular endothelial cells and extracellular matrix (ECM) proteins [7]. Early neovascularization after TEBG implantation in vivo is a major obstacle to overcome in achieving satisfactory healing $[8,9]$.

In our previous studies, TEBGs were prevascularized by inserting the femoral arteriovenous bundle and then implanted to treat large bone defects. At 4 and 8 weeks after implantation, significantly more new capillary vessels had formed inside prevascularized TEBGs than in control scaffolds [9-11]. However, the exact mechanisms for this positive effect remain unknown. Significant research has been conducted to explore the function, survival, and homing of exogenous seed cells [12-15]. However, endogenous cells play a more important role in the process of bone repair, yet there are few studies on the role and function of endogenous cells [16-18].

In this study, we elucidated the mechanisms by which transplantation of the femoral vascular bundle into the TEBG promotes angiogenesis and vascularization of the scaffold, specifically focusing on the participation of endogenous cells in bone defect repair. We established a large segmental femoral defect in $\mathrm{EGFP}^{+}$transgenic rats to investigate the recruitment and behavior of endogenous cells, by tracking the migration and survival of endogenous $\mathrm{EGFP}^{+}$cells within the scaffold using fluorescence microscopy; the scaffolds were seeded with wild-type cells isolated from $\mathrm{EGFP}^{-}$rats.

The $\beta$-TCP scaffold has spherical micropores with controllable size, and there are pore connections between the micropores to ensure that the connectivity rate is over 99\%. The pore size and porosity of the material were suitable for cell adhesion and tissue ingrowth. In addition, the scaffold has good biocompatibility and no carcinogenicity $[19,20]$.

We report that prevascularization led to the formation of a reticulated fibrin structure throughout the scaffold, which accelerated the recruitment of endogenous cells and type III collagen deposition by inducing high-level expression of connective tissue growth factor (CTGF) and, in turn, promoted the formation of new blood vessels by endogenous cells within the TEBG. This study provides a new basis for further clinical research to develop novel TEBG materials and approaches.

\section{Methods \\ Animals and ethics statement}

$\mathrm{EGFP}^{+}$transgenic (Sprague-Dawley (SD)) rats were purchased from Xing Ming Biomedical Technology Co., Ltd (Shanghai, China). Wild-type (WT, EGFP ${ }^{-}$) SD rats were obtained from the experimental animal center of Fourth Military Medical University, Xi'an, China. The rats were housed in a specific pathogen-free (SPF) animal facility at the animal center of Fourth Military Medical University.

All animal procedures were performed in the authorized animal care facility and were approved by the "Committee for the Care and Use of Laboratory Animals" of Fourth Military Medical University. All methods were performed in accordance with the relevant guidelines and regulations of the People's Republic of China.

\section{Preparation of TEBGs}

Porous $\beta$-tricalcium phosphate $(\beta$-TCP) scaffolds $(70 \%$ porosity, $4 \mathrm{~mm} \times 5 \mathrm{~mm}$ ) with a 1 -mm deep side groove were used to carry the implanted vascular bundles (Bio-lu Biomaterials, China). The $\beta$-TCP scaffold has spherical micropores with controllable size $(450 \pm 50 \mu \mathrm{m})$, and there are pore connections $(150 \pm 50 \mu \mathrm{m})$ between the micropores to ensure that the connectivity rate is over 99\% [19]. The mechanical strength is greater than $2 \mathrm{MPa}$ with no cytotoxicity and rejection.

EGFP $^{-}$BMSCs were isolated from the femoral bone marrow of 2-week-old wild-type rats. The culture medium was changed after the first $24 \mathrm{~h}$, and then every $48 \mathrm{~h}$ the medium was replaced to remove the dead and nonadherent cells. When the cell density reached approximately $80 \%$, the adherent cells were trypsinized, harvested, and seeded into new dishes at $1.0 \times 10^{5}$ cells. Third-passage BMSCs (after 3 weeks of culture) were seeded onto the scaffolds at a density of $5 \times 10^{6}$ cells $/ \mathrm{ml}$ and the cell-scaffold complexes (TEBGs) were incubated for 7 days before transplantation.

\section{Large segmental bone defect model in the rat femur}

The $\mathrm{EGFP}^{+}$SD rats (female, 12 weeks old, $220 \pm 10 \mathrm{~g}$ ) were randomly divided into two groups with eight rats in each group: the prevascularized TEBG group and the TEBG group. The animals were fasted overnight before surgery and anesthetized by intraperitoneal injection of $2 \% \mathrm{w} / \mathrm{v}$ pentobarbital sodium salt (45 mg/kg; Merck, Germany).

We made rat bone defect models as described previously [21]. Briefly, after being shaved and sterilized, the rat's femoral arteriovenous bundle was exposed by creating a $2-\mathrm{cm}$ incision inside the left hind limb. The arteriovenous bundle and saphenous nerve were then separated by microsurgical 
techniques, carefully dissociating out about $20-25 \mathrm{~mm}$. Next, blunt dissection of the fascia and muscle was performed to expose the left femoral shaft, and a large bone defect (5 $\mathrm{mm}$ in length) was created in the femur and fixed using an internal fixation plate. In the prevascularized TEBG group, we embedded the arteriovenous bundle into the $\beta$-TCP groove to construct a prevascularized TEBG complex, which was implanted into the defect site together. As a control, the TEBG group was implanted with $\beta$-TCP material at the defect site without embedding the vascular bundle.

The wounds were stitched and disinfected with iodophor. After the operation, X-ray imaging was performed at $35 \mathrm{kV}$ and $1.5 \mathrm{~mA}$ for $3 \mathrm{~s}$ using a Carestream DRX Ascend (Carestream Health, Canada) to check plate fixation and ensure successful establishment of the critical bone defect. After recovery, all experimental animals were intramuscularly injected with 400,000 $\mathrm{U}$ penicillin/day for 3 days.

In order to study the migration and distribution of endogenous cells and the survival of exogenous seed cells in the repair of bone defects, we used WT-BMSCs $\left(\mathrm{EGFP}^{-}\right)$as exogenous cells to generate a $\beta$-TCP complex for further implantation into $\mathrm{EGFP}^{+}$rats (all cells are EGFP $^{+}$). Cell sources in the TEBGs can be determined by tracing green fluorescent protein.

\section{Histological analysis}

At 1 and 4 weeks after operation, the femurs were carefully removed after cardiac perfusion and fixation, and secondary fixed in $4 \%$ paraformaldehyde (PFA) solution for 3 days. The samples were decalcified in 10\% ethylenediaminetetraacetic solution (EDTA; Sigma, USA) for 6 weeks, with gentle shaking at room temperature (until the samples were soft enough to be easily punctured by a needle).

The femoral specimens were dehydrated in a mixture of $10 \%$ gum arabic and $30 \%$ sucrose for 3 days at $4{ }^{\circ} \mathrm{C}$. After that, specimens were embedded in an OTC compound (Sakura Seiki, Tokyo, Japan) and serially sectioned perpendicular to the long axis of the femur using a cryostat (Jung CM3000; Leica, Wetzlar, Germany). Each section ( $8 \mu \mathrm{m}$ thick) was fixed on a glass slide and preserved at $-20^{\circ} \mathrm{C}$.

Some sections were rewarmed, stained with hematoxylin and eosin (H\&E), and observed by light microscopy (BX53; Olympus, Japan).

\section{Scanning electron microscopy analysis}

At 1 week post operation, the femurs were carefully removed and fixed in solution containing $4 \%$ paraformaldehyde (PFA) and 3\% glutaraldehyde (1:1) for 3 days. Inspection of the transversal surface of the material center (Hitachi-S3400N, Japan) used acceleration voltage $\geq 15 \mathrm{kV}$ and imaging distance $5-10 \mathrm{~mm}$.

\section{Immunofluorescence analysis}

A random selection of some sections from each group were rewarmed at $37{ }^{\circ} \mathrm{C}$ for $30 \mathrm{~min}$, wetted with PBS for 3 min, then permeabilized with $0.2 \%$ Triton X-100 (Sigma, USA) for $10 \mathrm{~min}$ at room temperature, washed three times with PBS, and incubated in blocking agent (10\% donkey serum; Solarbio) to block nonspecific binding. Sections were incubated with one of the primary antibody solutions-anti-fibrinogen (ab92572, 1:200; Abcam), anti-CTGF (ab6992, 1:200; Abcam), or anti-vWF (ab6994, 1:200; Abcam)-overnight at $4{ }^{\circ} \mathrm{C}$, rinsed thoroughly with PBS three times to remove unbound primary antibody, and then incubated with a secondary antibody of donkey anti-rabbit IgG H\&L (Alexa Fluor ${ }^{\circ}$ 594) (ab150076, 1:500; Abcam) for $1 \mathrm{~h}$ at $37^{\circ} \mathrm{C}$. Nuclei were stained with Hoechst 33342 (1:1000; Sigma) for $5 \mathrm{~min}$. The sections were disposed with Enhanced Antifade Mounting Medium (Leagene, China) and observed by laser scanning confocal microscopy (Nikon A1R, Japan).

\section{Sirius red staining}

The other frozen bone sections from each group were rewarmed and stained with sirius red (Solarbio, China) according to the manufacturer's protocol. Type I and type III collagen deposition were evaluated using a polarizing microscope imaging system (Leica, Germany). Type I collagen was colored orange or red and type III collagen was green under the polariscope.

\section{Statistical analysis}

Statistical analysis was performed using SPSS 22.0 software. Data were expressed as the mean \pm standard error of the mean (s.e.m.). Comparisons between the two groups were performed using independent-sample $t$ tests and correlation analyses. All data demonstrated a normal distribution and similar variation between groups. Statistical significance was defined as $P \leq 0.05$. All figures were generated using GraphPad Prism Software (Version 6; GraphPad Software, Inc., La Jolla, CA, USA).

\section{Results}

Implantation of a prevascularized TEBG scaffold into a large segmental bone defect in the femur of EGFP ${ }^{+}$rats A 5-mm defect of rat femur was regarded as a critical bone defect according to the previous study [22], which could not be repaired by itself. In our study, a $5-\mathrm{mm}$ critical-size bone defect model was established on the femoral shaft of $\mathrm{EGFP}^{+}$rats. In the prevascularized TEBG group, the femoral vascular bundle was inserted into the lateral groove of the scaffold (Fig. 1A). The TEBG group was only implanted into $\beta$-TCP as a control.

After operation, X-ray imaging confirmed the steel plates and screws were tightly fixed to the broken end of the bone defect, and the scaffolds did not exhibit slip, 


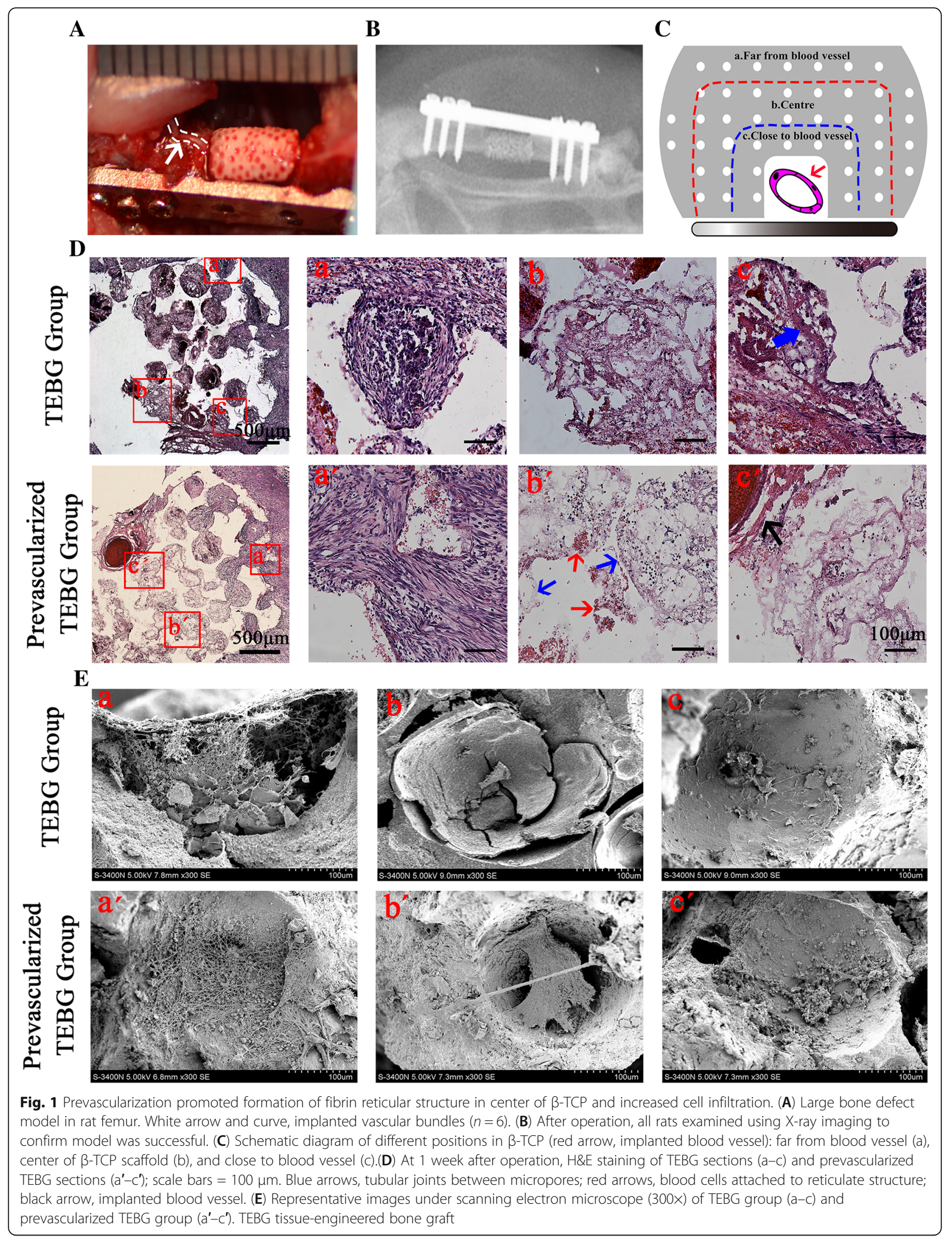


indicating the model was established successfully (Fig. 1B). Due to the tight fixation of steel plates, the injured limb of the experimental animal can bear the weight directly after awakening, without affecting their activities, diet, rest, and so on. In addition, the tissue ischemia caused by vascular injury could be compensated by collateral circulation, and hematoma and mild inflammation would last for 1-2 weeks after operation.

TEBGs were obtained at 1 and 4 weeks post operation, and tissue and cell infiltration were assessed in three different locations within the scaffold: far from the blood vessel $(>1200 \mu \mathrm{m}$; Fig. 1C, a), $\beta$-TCP center (600$1200 \mu \mathrm{m}$; Fig. $1 \mathrm{C}, \mathrm{b})$, and close to the blood vessel $(<$ $600 \mu \mathrm{m}$; Fig. 1C, c).

\section{Prevascularization promoted the formation of fibrin-like reticular structure in the center of $\beta$-TCP and increased cell infiltration}

H\&E staining and light microscopy were performed to assess the distribution of tissue and cells within the implants at 1 week post transplantation. Insertion of the vascular bundles into the scaffolds could be clearly observed in the prevascularized TEBG group.

The micropores of the prevascularization scaffolds were filled with reticular structure. In contrast, there were large blank areas in the center of the TEBG group (Fig. 1D). There were significant differences in cell status and extracellular matrix deposition at different locations of the two groups. A large number of inflammatory cells were located far from the vascular bundle in the TEBG control group, but the cells of the prevascularization TEBG group were arranged regularly and well in shape (Fig. 1D, a and a '). There were many fibrin-like reticulation and tubular connections between pores in the $\beta$-TCP center of the prevascularization group; simultaneously, a large number of blood cells attached to the network structure and the connecting tube, and these blood cells may provide oxygen for exogenous seed cells to increase their survival rate. On the contrary, there were some blank areas within the control sections, indicating that the extracellular matrix and cells had not yet infiltrated into the material center (Fig. 1D, b and b'). Reticular structures were also distributed around the vascular bundle in the prevascularized scaffolds. In contrast, cord-like structures created by the extension of the surrounding callus were observed around the edges of the control scaffolds (Fig. 1D, c and c '), but these cord-like structures only existed around the callus and did not extend to the center of the scaffolds. The results from the scanning electron microscope were consistent with the histopathological analysis mentioned earlier. There was almost no cell infiltration into the center of the TEBG group so that materials dry and crack (Fig. 1E, a-c). However, scanning electron microscopy (SEM) confirmed the reticulated, fibrin-like structure and that numerous blood clots were present throughout all three zones of the prevascularized scaffolds (Fig. 1E, $\mathrm{a}^{\prime}-\mathrm{c}^{\prime}$ ).

Surgical trauma causes blood vessel rupture, bleeding, and hematoma formation, and hematoma gradually transformed into a blood clot. New blood capillaries, fibroblasts, and phagocytic cells invaded the blood clot within $24 \mathrm{~h}$. At the same time, platelets, disintegrating tissue, and perivascular cells released some cytokines involved in bone repair, such as platelet-derived growth factor (PDGF), transforming growth factor (TGF- $\beta$ ), and so on [23, 24]. Clots would not always exist and are gradually cleared in later stages and quickly became granulation tissue, which in turn formed fibrous callus [25]. Therefore, in the early stage of bone defect, there would be some blood clots blocking the scaffold's pores. However, with the destruction of erythrocytes in the hematoma, fibrin exudes and blood clots were replaced by a fibrin network structure. Thus, our data indicated that prevascularization promoted formation of a fibrin network structure within the scaffolds, which provided a support and bridge for endogenous cell migration between the micropores of the material.

\section{Prevascularization increased the expression of fibrinogen, and then promoted $\mathrm{EGFP}^{+}$endogenous cell infiltration}

In order to confirm whether insertion of the vascular bundle promoted fibrin production, we examined the expression of fibrinogen by immunofluorescence staining (Fig. 2A, B). The relative fluorescence integrated optical density (IOD) of fibrinogen in the prevascularization TEBG group was significantly higher than in the TEBG group in all three zones of the scaffold: close to and far from the vascular bundle, and especially in the center of $\beta$-TCP. Thus, the immunofluorescence staining confirmed the histopathology and SEM observations that prevascularization promoted formation of a reticular fibrin network within the materials.

In this model, it was possible to distinguish between endogenous cells $\left(\mathrm{EGFP}^{+}\right)$and exogenous cells $\left(\mathrm{EGFP}^{-}\right)$ by tracing the green fluorescent protein markers. Thus, we explored whether this reticular structure promoted infiltration of endogenous cells and survival of exogenous seed cells. At 1 and 4 weeks post operation, prevascularization can significantly increase the total number of cells in the materials (Fig. 2C-E). At 1 week, the number of endogenous cells in the prevascularized group was more than twofold higher than the number of cells in the TEBG group. However, the proportion of $\mathrm{EGFP}^{+}$endogenous cells in the total number of cells was lower than that of the control group, indicating that vascular bundle implantation significantly promoted the survival of exogenous seed cells (Fig. 2E).

Collectively, these analyses indicated that the fibrin network within the prevascularized scaffolds provided a 


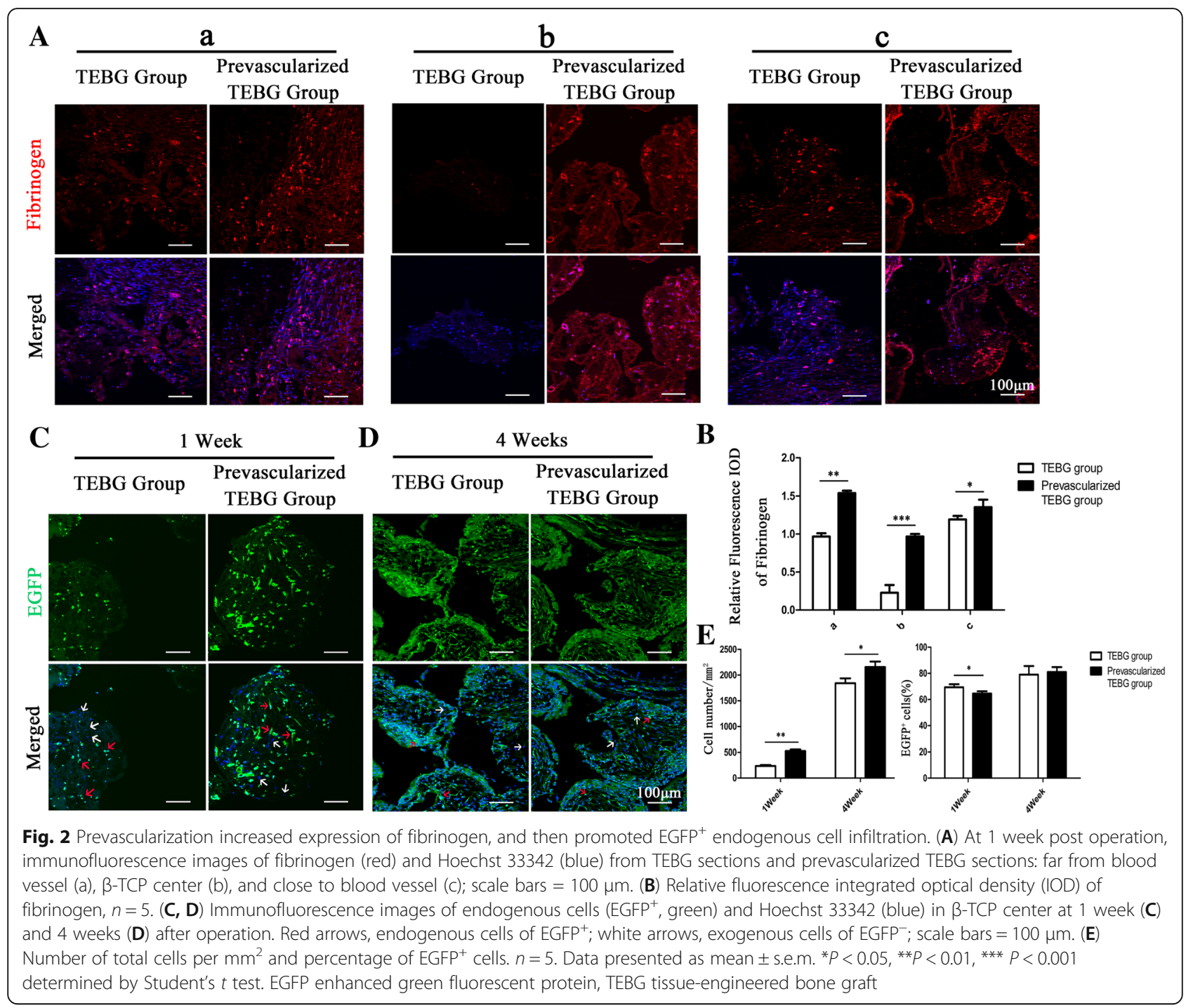

structural connection between the internal micropores of the scaffold, which supported endogenous cell infiltration and migration, which may facilitate formation of a vascular network to supply nutrients and oxygen to the exogenous seed cells and increase their survival rate.

\section{Prevascularization increased the expression of CTGF}

CTGF is a modular secreted protein closely associated with multiple cellular events such as chondrogenesis, skeletogenesis, trauma repair, and angiogenesis [26]. Under physiological conditions, CTGF appears to have a role in collagen synthesis, and to accelerate the production of extracellular matrix and support the newly formed vascular structure to promote angiogenesis [27]. Therefore, we assessed the expression of CTGF at 1 week after implantation by immunofluorescent analysis of frozen sections. The results showed that prevascularization significantly increased the distribution areas and relative IOD of
CTGF in all three zones of the grafts (Fig. 3A, B). A high level of CTGF expression is likely to facilitate recruitment of cells, and thus CTGF may promote infiltration of endogenous cells into tissue-engineered bone grafts, to promote angiogenesis and accelerate bone repair.

\section{Prevascularization enhanced the deposition of collagen type I/III within the scaffold}

Collagen type I is the main component of bone tissue, contributing to the elasticity and toughness of bone. Collagen type III is a reconstituted collagen that plays an important role in tissue damage repair. It can directly promote angiogenesis and maintain the function of capillaries.

Our results indicated that vascular bundle implantation increased endogenous cell infiltration and exogenous cell survival. Thus, we explored whether these cells within the scaffold secrete matrix and participated in tissue reconstruction. Sirius red staining showed no obvious collagen 


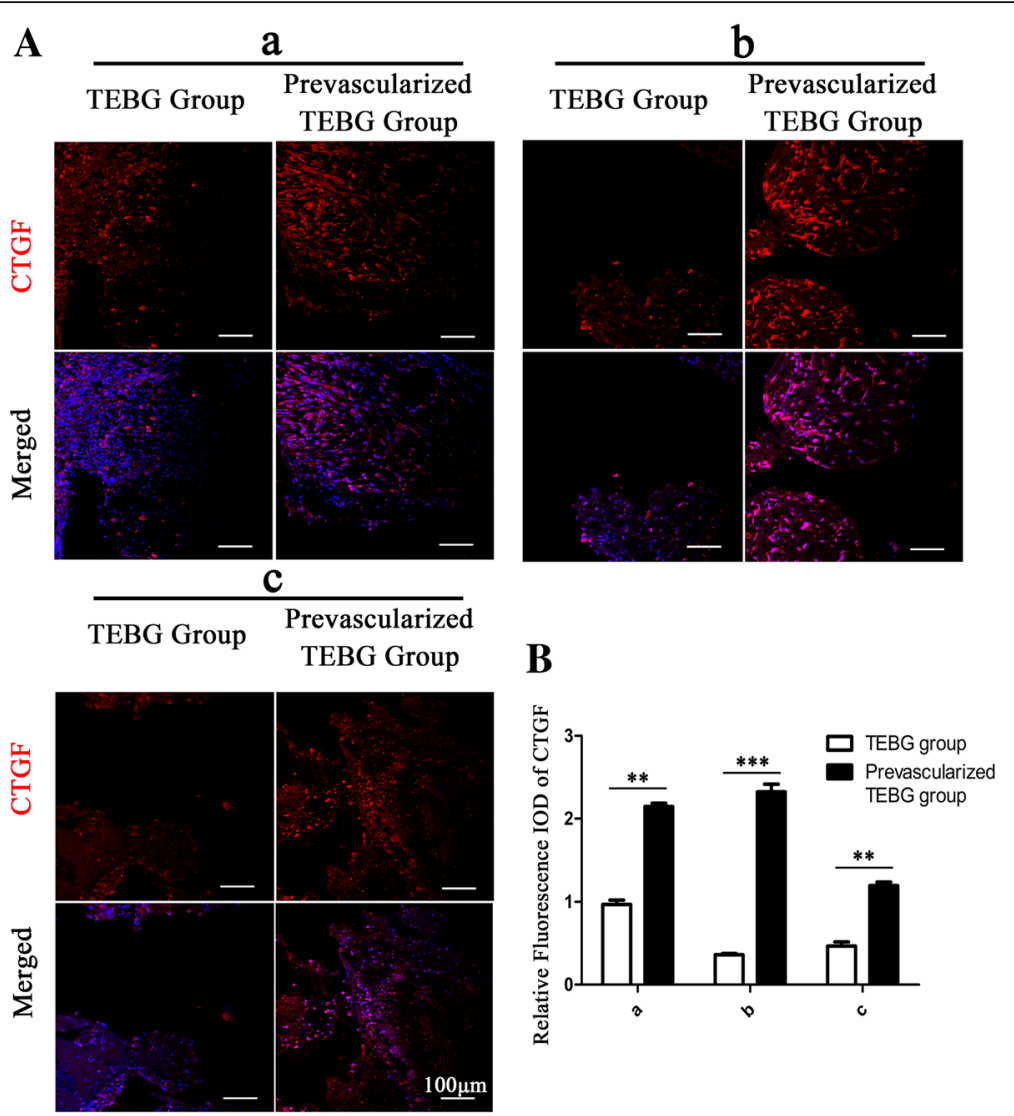

Fig. 3 Prevascularization increased expression of CTGF. (A) At 1 week post operation, immunofluorescence images of CTGF (red) and Hoechst 33342 (blue) from TEBG sections and prevascularized TEBG sections: far from blood vessel (a), center (b), and close to blood vessel (c); scale bars $=100 \mu \mathrm{m}$. (B) Relative fluorescence integrated optical density (IOD) of CTGF, $n=5$. Data presented as mean \pm s.e.m. ${ }^{* *} P<0.01,{ }^{* * *} P<0.001$ determined by Student $t$ test. CTGF connective tissue growth factor, TEBG tissue-engineered bone graft

deposition within the two groups at 1 week after implantation. Interestingly, a high level of type I collagen expression was observed around the implanted vascular bundle in the prevascularized TEBG group (Fig. 4A).

At 4 weeks after the operation, prevascularized scaffolds contained higher levels of both collagen type I and type III close to the vascular bundle, far from the vascular bundle, and in the center of the scaffold compared to control scaffolds (Fig. 4B, C). In addition, the proportion of type III collagen relative to total collagen was significantly higher in prevascularized TEBG scaffolds than in TEBG scaffolds.

Dynamic interactions between extracellular matrix (ECM) and growth factors are integral to wound healing. These interactions take several forms that may be categorized as direct or indirect. Collagen III is one of the ECM components that can directly bind to or release certain growth factors. Additionally, the collagen can bind to cell surface receptors in the cytokine, chemokine, or growth factor families and stimulate cellular activities [28]. Collagen III has been widely used to facilitate cell growth and differentiation in constructive remodeling [29]. In summary, collagen III interacts with various cells and growth factors, which enhances communication and interaction between cells and accelerates bone defect repair.

Prevascularization promoted formation of $\mathrm{vWF}^{+}$capillarylike tubular connections between the scaffold micropores The connection between micropores in porous materials plays a crucial role in tissue repair and material degradation, such as supporting cell adhesion, migration, and interaction, and promoting matrix deposition. $\mathrm{H} \& \mathrm{E}$ staining suggested that the reticulated fibrin structure promoted cell migration and construction of tubular connections between the pores within $\beta$-TCP (Figs. 1D and 5a). SEM revealed that numerous blood cells were attached to the tubular structure (Fig. 5b), in accordance with the histological staining (Fig. 1D, b'). Further study found high expression of CTGF in the tubular area at 1 week after surgery (Fig. $5 \mathrm{c}$ ), which promoted the recruitment of increased numbers of endogenous cells to this region, facilitating cellular communication and delivery of nutrients between the micropores. Furthermore, the recruited cells secreted type III collagen that filled the tubular structure (Fig. 5d), and eventually transformed into $\mathrm{vWF}^{+}$capillary-like structure at 4 weeks 


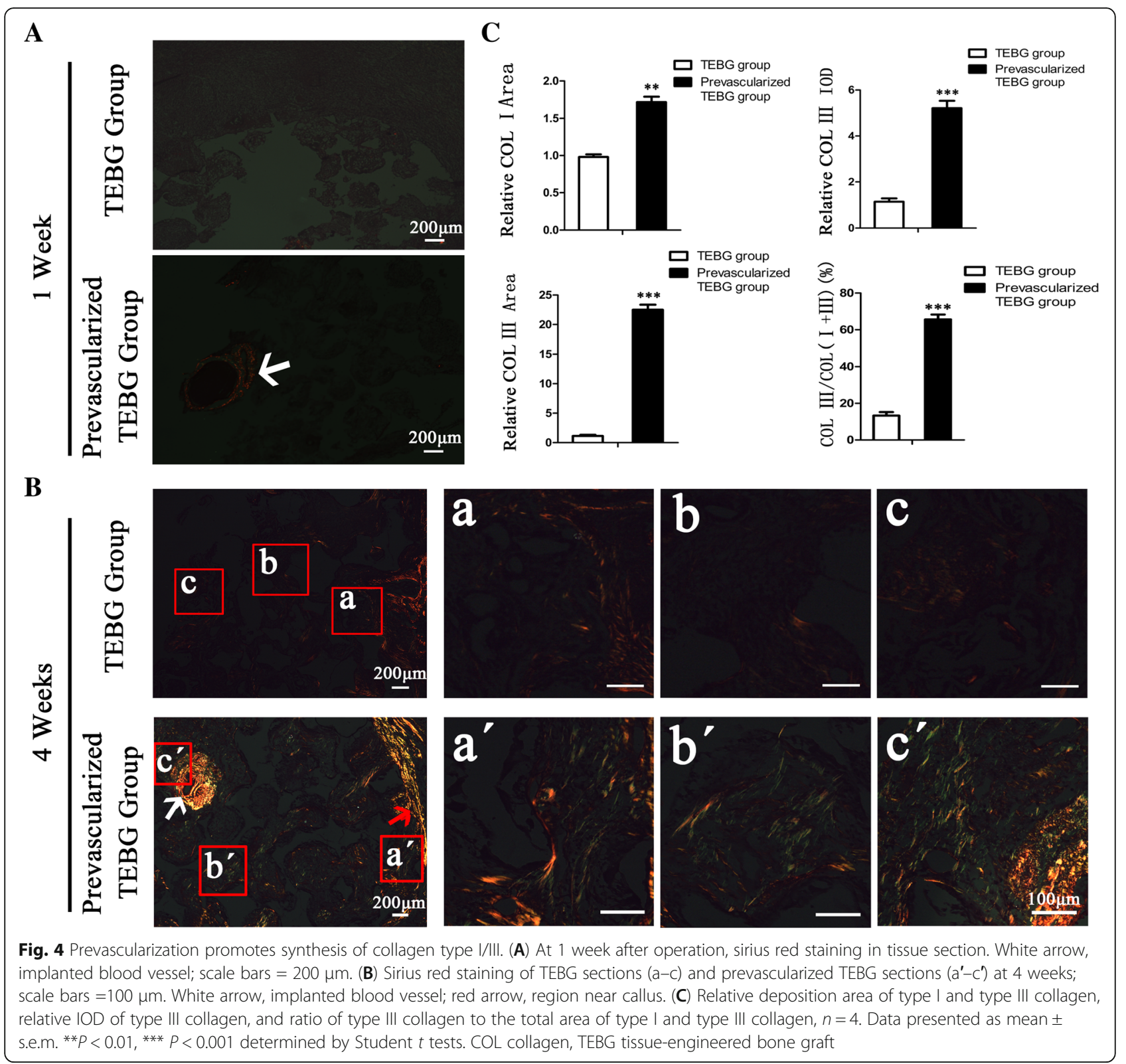

after implantation (Fig. 5e). In addition, the diameter of these $\mathrm{vWF}^{+}$structures was $5-10 \mu \mathrm{m}$, which coincided with the diameter of capillaries [30-32].

These results suggested that the tubular structure of fibrin, which is gradually transformed into a vascular structure due to the action of factors such as CTGF and type III collagen, and the tubular fibrin network provided a bridge to the connect the micropores and promote vascularization of $\beta$-TCP.

\section{Prevascularization promoted the angiogenesis of endogenous cells}

Endogenous cells play a more important role in tissue damage repair than exogenous cells. We explored the effect of prevascularization on endogenous cell-mediated angiogenesis by fluorescence colocalization of EGFP and vWF. At 1 week post operation, prevascularization can significantly increase the number of endogenous cells in $\mathrm{vWF}^{+}$(Fig. 6a, b), which may be involved in tissue reconstruction and angiogenesis. At 4 weeks after surgery, $\mathrm{vWF}^{+}$tubular structures were present between the micropores of the material. The number of these capillary-like structures in the prevascularization group was significantly higher than in the TEBG group. However, there was no obvious difference in capillary length (Fig. 6a, c), which may be related to the relatively uniform distance between the micropores of the material. 


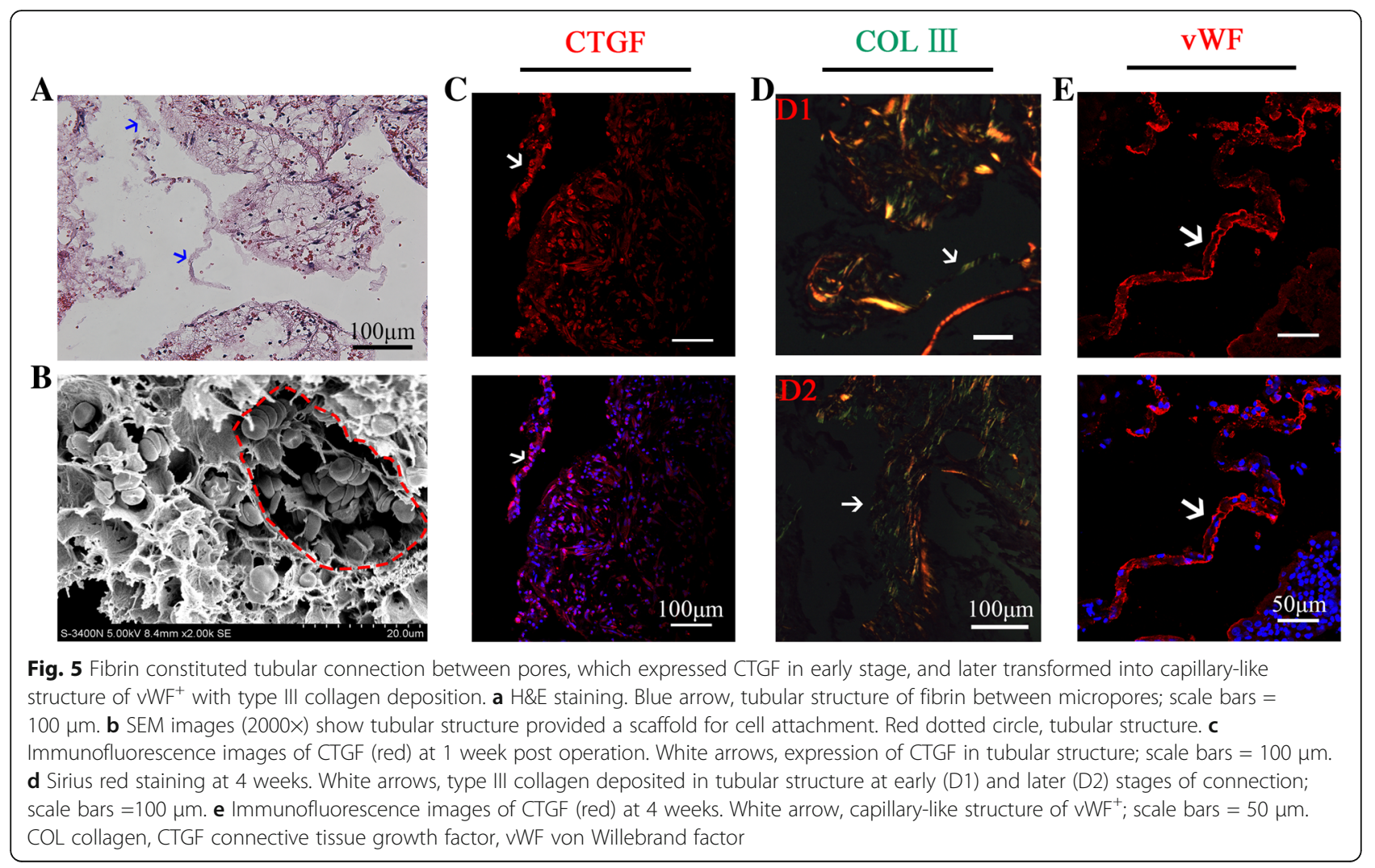

\section{Prevascularization promoted bone regeneration}

At 4 weeks after surgery, prevascularization significantly promoted tissue ingrowth and material degradation. At 8 weeks, there was obvious new bone formation at the edge of material pores (Additional file 1: Figure S1A). H\&E staining, X-ray, and micro-CT results showed that vascular bundle implantation significantly accelerated the process of bone repair (Additional file 1: Figure S1B, C). In addition, immunofluorescence staining showed that the number of osteoblasts $\left(\right.$ Osterix $\left.^{+}\right)$in the prevascularization group was significantly higher than in the TEBG control group (Additional file 1: Figure S1D, E). It is interesting that both endogenous and exogenous cells (BMSCs) are involved in the process of bone regeneration (Additional file 1: Figure S1D).

In summary, the results of this study showed that in the early stage of vascular bundle implantation, the content of fibrinogen increased, forming a fibrin reticular structure and a connecting tube between micropores, providing scaffolds for cell attachment and migration. Meanwhile, CTGF expression is increased, and more endogenous cells such as vascular endothelial cells can be recruited to participate in bone defect repair (Fig. 7, left panel). In the later stage, these tubular connections transformed into $\mathrm{vWF}^{+}$capillary-like structures with type III collagen deposition, and promoted neovascularization by endogenous cells (Fig. 7, right panel). These nascent capillaries provided nutritional support to maintain cell survival and function, thereby accelerating the process of bone defect repair.

\section{Discussion}

We reported previously that TEBGs prevascularized by inserting a vascular bundle significantly increased the number of capillary vessels. In this study, we aimed to identify the mechanism by which prevascularization promotes vascularization of the TEBG.

Since the 1990s, tissue engineering has undergone many advances in scaffold development, seeding cells, animal models, and clinical trials [33, 34]. Some previous studies have shown that endogenous cells are the main contributors to tissue reconstruction and angiogenesis; they play a more important role than exogenous cells in the process of defect repair [17, 18, 35]. However, most studies have focused on the survival and function of exogenous seed cells, with little attention given to the distribution and behavior of endogenous cells. Our study successfully generated a 5-mm-length defect in rat femurs using customized internal fixation plates. With the use of $\mathrm{EGFP}^{+}$transgenic rats, this large defect model provided the possibility to further explore the contribution of endogenous cells and exact molecular mechanisms by tracing EGFP. 


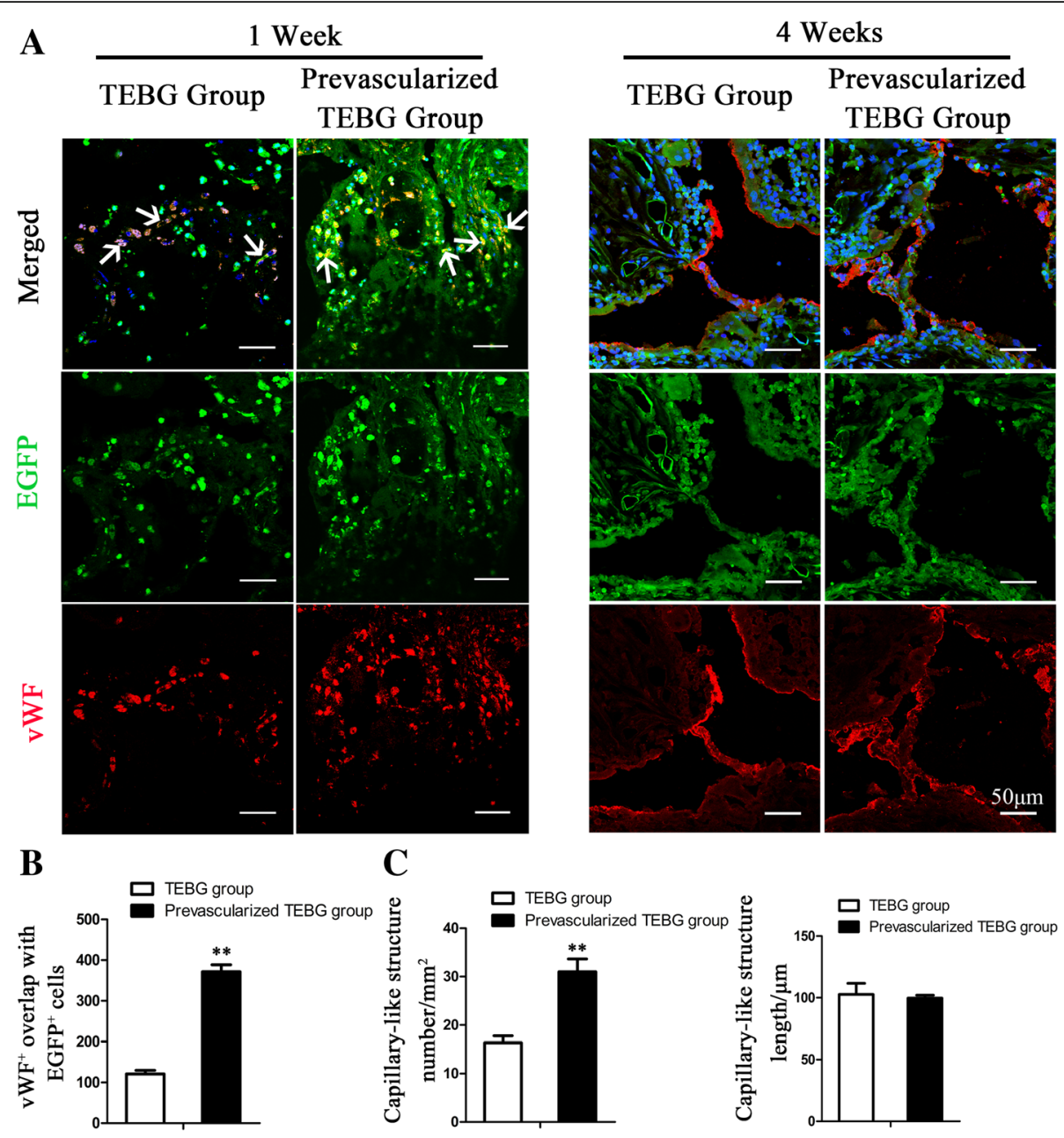

Fig. 6 Prevascularization increased number of $\mathrm{VWF}^{+}$endogenous cells and neonatal capillary-like structures. a Immunofluorescence images of VWF (red) and EGFP (green) from TEBG sections and prevascularized TEBG sections at 1 and 4 weeks after transplantation. White arrows, doublepositive staining cells. Hoechst 33342 stained nuclei blue; scale bars $=50 \mu \mathrm{m}$. b Number of $\mathrm{VWF}^{+}$cells overlaid with EGFP ${ }^{+}$endogenous cells, $n=6$. c Number of capillary-like structures $\left(\mathrm{mm}^{2}\right)$ and length $(\mu \mathrm{m}), n=6$. Data presented as mean \pm s.e.m. ${ }^{* *} P<0.01$ determined by Student $t$ tests. EGFP enhanced green fluorescent protein, TEBG tissue-engineered bone graft vWF von Willebrand factor

Our observations indicated that insertion of the vascular bundle into the scaffold before implantation led to accumulation of fibrinogen within the TEBG. Fibrinogen, a major plasma protein that plays a key role in hemostasis, is converted to fibrin in response to bone injury $[36,37]$, and the resulting reticulated fibrin network created cross-linked, tube-like connections between the micropores of the scaffold at 1 week after implantation. This fibrin network appeared to provide a substrate for cell adhesion to facilitate exogenous seed cell survival and endogenous cell migration into the scaffold. Histology, SEM, and EGFP fluorescence tracing confirmed that endogenous cells infiltrated into the TEBGs immediately after implantation. Moreover, the fibrin network promoted exogenous seed cell survival and also significantly increased the number of endogenous cells that migrated and/or proliferated within the scaffold.
(Fig. 2C-E). Similarly, recent articles have reported that fibrinogen scaffolds significantly promote vascularization in vivo $[38,39]$.

Fibrin polymers are widely used in the tissue engineering field as biomaterials. The fibrinogen content of fibrin glue/bone powder scaffolds would be benefit bone tissue engineering [40]. Combining platelet-rich fibrin (PRF) with the synthetic material (the combination of hydroxyapatite and $\beta$-TCP) resulted in more cortical and subcortical bone formation [41]. Fibrin preparations can support the proliferation and growth of periosteal cells to form well-combined active biological materials [42]. Therefore, fibrin could be clinically applicable in bone regeneration therapy.

In addition, prevascularization induced high-level CTGF expression within the scaffolds at 1 week after implantation. CTGF (also known as CCN2), a cysteine-rich protein that 


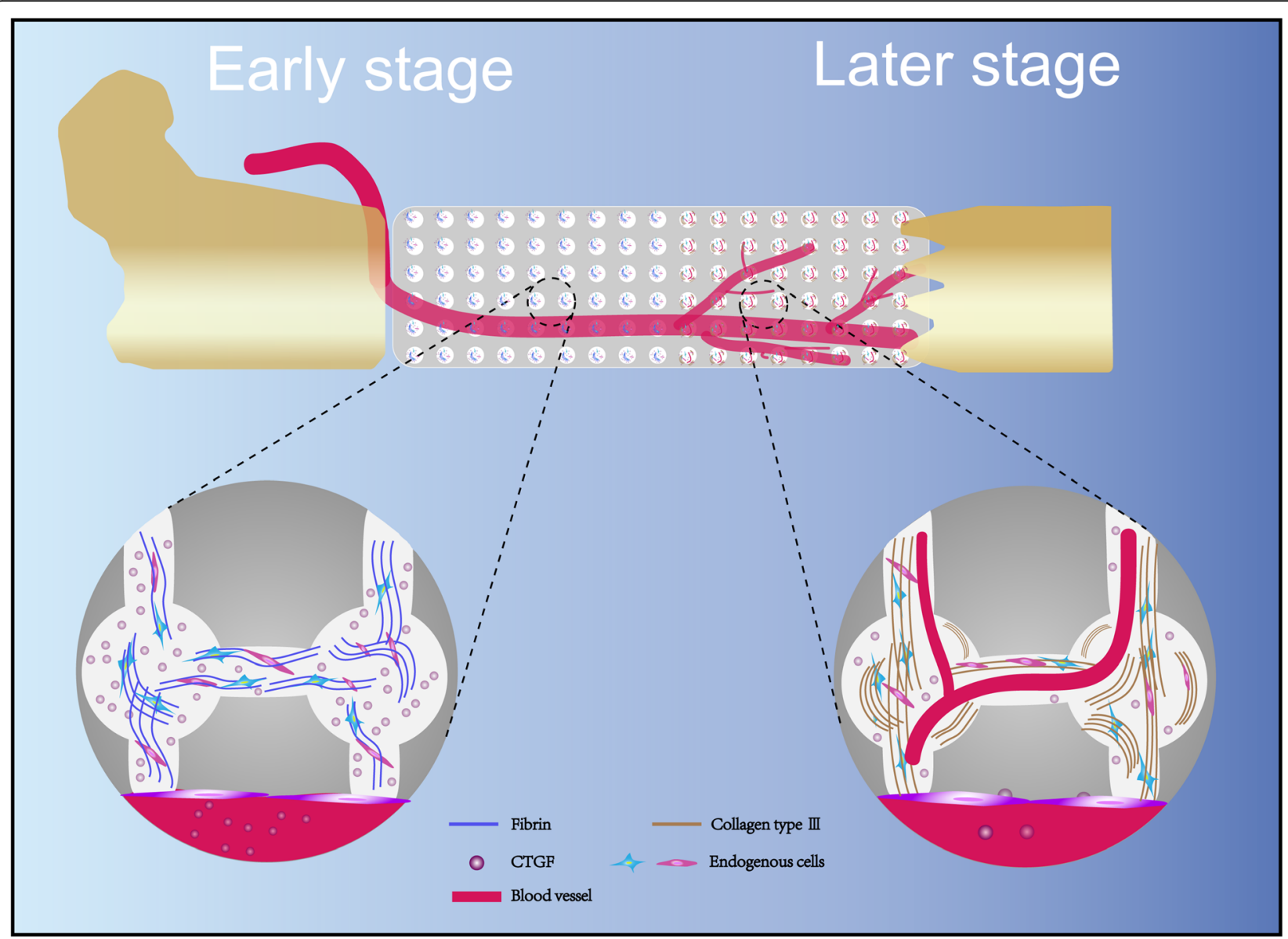

Fig. 7 Prevascularization promoted endogenous cell-mediated angiogenesis in TEBG. Fibrin content increased at early stage of vascular implantation, and reticular formation of fibrin provided scaffold for endogenous cell infiltration and created a tubular connection between pores. In addition, prevascularization improved expression of CTGF, and then promoted collagen synthesis and angiogenesis (left panel). In the later stage, these tubular connections transformed into $\mathrm{WWF}^{+}$capillary-like structures with type III collagen deposition, eventually increasing number of new blood vessels (right panel). CTGF connective tissue growth factor

is widely expressed in response to tissue damage, can be synthesized by fibroblasts, smooth muscle cells, and endothelial cells. CTGF has been shown to promote cell adhesion, migration, and proliferation [26]. CTGF exerts proangiogenic activity and interacts with other proangiogenic proteins via several mechanisms to regulate angiogenesis [27], and has also been reported to promote tumor angiogenesis [43, 44] Liu et al. [45] suggested that CTGF significantly increased angiogenesis regulated by vascular endothelial growth factor (VEGF) in human synovial fibroblasts. Grote et al. [46] showed that CTGF recruited CD34 ${ }^{+}$ progenitor cells and thereby promotes endothelial cell proliferation and capillary formation.

CTGF also promoted collagen synthesis [47-49], which may explain the increased deposition of type I and type III collagen in the prevascularized scaffolds at 4 weeks post operation (Fig. 4B). The components of the ECM, such as collagen type I and III, play vital roles in the formation of new blood vessels [50, 51]. Type I collagen is the main component of bone tissue, and type III collagen is expressed at high levels during remodeling of injured tissue [50, 52]. Type III collagen is also abundant in blood vessels, where it contributes to the strength and elasticity of capillaries.

Fibrin and collagen differentially, but synergistically, regulate angiogenesis [7]. The fibrin network that formed between the pores of the scaffold expressed CTGF at 1 week after implantation, and had transformed into $\mathrm{vWF}^{+}$capillary-like structures with deposition of type III collagen by 4 weeks after implantation (Fig. 5), which in turn promoted neovascularization by endogenous cells (Fig. 7, right panel). Fluorescence microscopy confirmed that prevascularization promoted angiogenesis mediated by endogenous cells within the TEBGs. These nascent capillaries are likely to provide nutritional support to maintain cell survival and function, and thereby accelerate bone defect repair processes. Overall, this study suggests prevascularization induces angiogenesis within the scaffold 
via a mechanism involving fibrinogen/fibrin-CTGF-type III collagen, as illustrated in Fig. 7.

The exogenous cells used in this experiment were purified BMSCs $\left(\mathrm{CD} 90^{+}, \mathrm{CD} 34^{-}, \mathrm{CD} 45^{-}, \mathrm{CD} 11 \mathrm{~b} / \mathrm{c}^{-}\right)$, which could differentiate into osteoblasts, cartilage, and adipocytes in vitro [21, 34]. BMSCs have low immunogenicity and can induce a transient immunoreaction in early stage after transplantation, whereas the long-term engineered bone formation was not affected [53, 54]. Moreover, BMSCs induce T-cell hyporesponsiveness and prolong graft survival in the rat vascularized composite allotransplantation model. BMSCs exhibit immunomodulatory properties against acute rejection that can be realized without the need for significant recipient immunosuppression [55].

Correspondingly, no obvious rejection was found in our experimental animals, and only mild inflammation would last for 1-2 weeks after surgery, which may be caused by surgical trauma. In addition, inflammation in the prevascularization group was lower than in the TEBG group.

One limitation of this study is that all endogenous cells expressed EGFP. Therefore, the key role of specific cells is unknown. Further in-vitro and in-vivo experiments should be designed to further explore the precise roles and molecular mechanisms of action of individual types of endogenous. Furthermore, we divided the grafts into three regions: far from the vascular bundle, close to the vascular bundle, and the material center. The results showed that the histopathological morphology, inflammation levels, and repair process varied at different locations, but the mechanism for such a significant difference still needs further study.

In summary, this exploration of the effect of prevascularization on endogenous cells provided a new perspective for the study of TEBG vascularization, which may also help to develop more molecular-based drugs and materials, thus replacing implantation of the vascular bundle and increasing the efficiency of neovascularization within TEBGs.

\section{Conclusions}

The contribution of endogenous cells in tissue reconstruction and functional regeneration of bone injury is greater than that of exogenous seed cells. How to improve the infiltration speed and quantity of endogenous cells is important for bone and blood vessel regeneration. In this paper, EGFP transgenic rats were used to establish a model of large femoral bone defect. For the first time, we found that the implantation of vascular bundles could increase the expression of fibrinogen and CTGF, and then the fibrin reticular scaffold formed by fibrinogen significantly increased the number of vascular endothelial cells and other endogenous cells. Finally, with the deposition of type III collagen, a more capillary-like structure of $\mathrm{vWF}^{+}$was formed within the material, thus promoting vascularization of the TEBGs. In addition, this molecular mechanism can be used to establish fast-acting angiogenesis materials in future clinical applications.

\section{Additional file}

\begin{abstract}
Additional file 1: Figure S1. Prevascularization promoted bone regeneration in TEBG. (A) H\&E staining of TEBG sections and prevascularized TEBG sections at 4 weeks and 8 weeks post operation. Black arrow, implanted blood vessel. Red arrows, regenerative bone tissue. (B) At 8 weeks, X-ray imaging analysis of bone defect repair and micro-CT 3D reconstruction images of new bone formation. (C) BV/TV used to evaluate new bone formation. ${ }^{*} P<0.01$ determined by Student $t$ tests. (D) Immunofluorescence images of Osterix (red) and EGFP (green) from TEBG sections and prevascularized TEBG sections at 8 weeks after transplantation. Hoechst 33342 stained nuclei blue; scale bars $=50 \mu \mathrm{m}$. Red arrows, endogenous osteoblasts; white arrows, exogenous-derived osteoblasts; scale bars $=20 \mu \mathrm{m}$. (E) Total number of Osterix ${ }^{+}$cells. ${ }^{* *} P<$ 0.01 determined by Student $t$ tests. (TIF $9451 \mathrm{~kb}$ )
\end{abstract}

\section{Funding}

This work was supported by National Natural Science Foundation of China (81430049, 81572192, and 2017SF-014).

Availability of data and materials

All supporting data are included in the article and its Additional files.

\section{Authors' contributions}

$P C$ and $L Y$ designed the research. $P C$ and $D L$ performed the research. PC, $L Y$, $\mathrm{TC}$, and $C W$ analyzed the data. $P C$ and $Y G$ wrote the paper. DL, PC, HJ, JW,

$J$, SZ, YS, and BL created the rat bone defect models. LY and GP conceived

of the study, reviewed the data, and approved the final version of the

manuscript. All authors read and approved the final manuscript.

\section{Ethics approval and consent to participate}

All procedures on rats were performed in the authorized animal care facility and were approved by the "Committee for the Care and Use of Laboratory Animals" of Fourth Military Medical University. All methods were performed in accordance with the relevant guidelines and regulations of the People's Republic of China.

\section{Consent for publication}

All authors consent to publication of the present manuscript.

\section{Competing interests}

The authors declare that they have no competing interests.

\section{Publisher's Note}

Springer Nature remains neutral with regard to jurisdictional claims in published maps and institutional affiliations.

\section{Author details}

${ }^{1}$ Institute of Orthopedic Surgery, Xijing Hospital, Fourth Military Medical University, Xi'an 710032, People's Republic of China. ${ }^{2}$ Hospital 463 of People's Liberation Army, Shenyang 110042, People's Republic of China. ${ }^{3}$ Department of Orthopedics, The 251st Hospital of PLA, Zhangjiakou 075000, China.

Received: 27 March 2018 Revised: 29 May 2018

Accepted: 13 June 2018 Published online: 04 July 2018

\section{References}

1. Muscolo DL, Ayerza MA, Aponte-Tinao LA. Massive allograft use in orthopedic oncology. Orthop Clin North Am. 2006;37(1):65-74.

2. Myeroff $C$, Archdeacon M. Autogenous bone graft: donor sites and techniques. J Bone Joint Surg Am. 2011;93(23):2227-36. 
3. Bi L, Cheng W, Fan H, Pei G. Reconstruction of goat tibial defects using an injectable tricalcium phosphate/chitosan in combination with autologous platelet-rich plasma. Biomaterials. 2010;31(12):3201-11.

4. Rezwan K, Chen QJ, Boccaccini AR. Biodegradable and bioactive porous polymer/inorganic composite scaffolds for bone tissue engineering. Biomaterials. 2006:27(18):3413-31.

5. Khan Y, Yaszemski MJ, Mikos AG, Laurencin CT. Tissue engineering of bone: material and matrix considerations. J Bone Joint Surg American. 2008; 90(Suppl 1):36-42.

6. Marcacci M, Kon E, Moukhachev V, Lavroukov A, Kutepov S, Quarto R, Mastrogiacomo M, Cancedda R. Stem cells associated with macroporous bioceramics for long bone repair: 6- to 7-year outcome of a pilot clinical study. Tissue Eng. 2007;13(5):947-55.

7. Feng $X$, Tonnesen MG, Mousa SA, Clark RAF. Fibrin and collagen differentially but synergistically regulate sprout angiogenesis of human dermal microvascular endothelial cells in 3-dimensional matrix. Int J Cell Biol. 2013;2013:1-11.

8. Lovett M, Lee K, Edwards A, Kaplan DL. Vascularization strategies for tissue engineering. Tissue Eng Part B Rev. 2009;15(3):353-70.

9. Wang L, Fan H, Zhang Z, Lou A, Pei G, Jiang S, Mu T, Qin J, Chen S, Jin D. Osteogenesis and angiogenesis of tissue-engineered bone constructed by prevascularized $\beta$-tricalcium phosphate scaffold and mesenchymal stem cells. Biomaterials. 2010;31(36):9452-61.

10. Fan $H$, Zeng $X$, Wang $X$, Zhu R, Pei G. Efficacy of prevascularization for segmental bone defect repair using $\beta$-tricalcium phosphate scaffold in rhesus monkey. Biomaterials. 2014;35(26):7407-15.

11. Fan J, Mu T, Qin J, Bi L, Pei G. Different effects of implanting sensory nerve or blood vessel on the vascularization, neurotization, and osteogenesis of tissue-engineered bone in vivo. Biomed Res Int. 2014;2014:1-10.

12. Stephan SJ, Tholpady SS, Gross B, Petrie-Aronin CE, Botchway EA, Nair LS, Ogle RC, Park SS. Injectable tissue-engineered bone repair of a rat calvarial defect. Laryngoscope. 2010;120(5):895-901.

13. Zhang $W$, Zhu $C$, Ye $D, X u L$, Zhang $X$. Porous silk scaffolds for delivery of growth factors and stem cells to enhance bone regeneration. PLoS One. 2014;9(7):e102371.

14. Monteiro BS, Del Carlo RJ, Argôlo-Neto NM, Nardi NB, Carvalho PH, Bonfá Lde P, Chagastelles PC, Moreira HN, Viloria MI, Santos BS. Association of mesenchymal stem cells with platelet rich plasma on the repair of critical calvarial defects in mice 1. Acta Cir Bras. 2012;27(3):201-9.

15. Monteiro BS, Lo-Neto NA, Nardi NB, Chagastelles PC, Carvalho PH. Treatment of critical defects produced in calvaria of mice with mesenchymal stem cells. An Acad Bras Cienc. 2012;84(3):841-51.

16. Herrmann M, Verrier S, Alini M. Strategies to stimulate mobilization and homing of endogenous stem and progenitor cells for bone tissue repair. Front Bioeng Biotechnol. 2015;3:79.

17. Chen J, Zhang Y, Pan P, Fan T, Chen M. In situ strategy for bone repair by facilitated endogenous tissue engineering. Colloids Surf B Biointerfaces. 2015;135:581-7.

18. Sarmento B, Neves JD, Sarmento B, Das Neves J. Chitosan-based systems for biopharmaceuticals: delivery, targeting and polymer therapeutics. Chichester, West Sussex: Wiley; 2012.

19. Xiao X, Wang W, Liu D, Zhang H, Gao P, Geng L, Yuan Y, Lu J, Wang Z. The promotion of angiogenesis induced by three-dimensional porous betatricalcium phosphate scaffold with different interconnection sizes via activation of PI3K/Akt pathways. Sci Rep. 2015;5:9409.

20. Feng $B$, Jinkang $Z$, Zhen W, Jianxi L, Jiang C, Jian L, Guolin $M$, Xin D. The effect of pore size on tissue ingrowth and neovascularization in porous bioceramics of controlled architecture in vivo. Biomed Mater. 2011;6(1):015007.

21. Jiang $H$, Cheng $P$, Li D, Li J, Wang J, Gao Y, Zhang S, Cao T, Wang C, Yang L, et al. Novel standardized massive bone defect model in rats employing an internal eight-hole stainless steel plate for bone tissue engineering. J tissue Eng Regen Med. 2018;12(4):e2162-71.

22. Harada N, Watanabe $Y$, Sato K, Abe S, Yamanaka K, Sakai Y, Kaneko T, Matsushita T. Bone regeneration in a massive rat femur defect through endochondral ossification achieved with chondrogenically differentiated MSCs in a degradable scaffold. Biomaterials. 2014;35(27):7800-10.

23. Wang X, Zhang Y, Ji W, Ao J. Categorising bone defect hematomas-enhance early bone healing. Med Hypotheses. 2018;113:77-80.

24. Weisel JW, Litvinov RI. Mechanisms of fibrin polymerization and clinical implications. Blood. 2013;121(10):1712-9.

25. Schmidt-Bleek K, Schell H, Lienau J, Schulz N, Hoff P, Pfaff M, Schmidt G, Martin C, Perka C, Buttgereit F, et al. Initial immune reaction and angiogenesis in bone healing. J Tissue Eng Regen Med. 2014;8(2):120-30.
26. Mendes FA, Coelho Aguiar JM, Kahn SA, Reis AH, Dubois LG, Romão LF, Ferreira LSS, Chneiweiss H, Moura Neto V, Abreu JG. Connective-tissue growth factor (CTGF/CCN2) induces astrogenesis and fibronectin expression of embryonic neural cells in vitro. PLoS One. 2015;10(8):e133689.

27. Ponticos M. Connective tissue growth factor (CCN2) in blood vessels. Vasc Pharmacol. 2013;58(3):189-93.

28. Schultz GS, Wysocki A. Interactions between extracellular matrix and growth factors in wound healing. Wound Repair Regen. 2009;17(2):153-62.

29. Chan EC, Kuo S, Kong AM, Morrison WA, Dusting GJ, Mitchell GM, Lim SY, Liu G. Three dimensional collagen scaffold promotes intrinsic vascularisation for tissue engineering applications. PLoS One. 2016;11(2):e149799.

30. Sakita M, Murakami S, Fujino H. Age-related morphological regression of myelinated fibers and capillary architecture of distal peripheral nerves in rats. BMC Neurosci. 2016;17(1):39.

31. Zong Y, Zhou X, Cheng J, Yu J, Wu J, Jiang C. Cannabinoids regulate the diameter of pericyte-containing retinal capillaries in rats. Cell Physiol Biochem. 2018:43(5):2088-101.

32. Tata DA, Anderson BJ. A new method for the investigation of capillary structure. J Neurosci Methods. 2002;113(2):199-206.

33. Chen F, Liu X. Advancing biomaterials of human origin for tissue engineering. Prog Polym Sci. 2016;53:86-168.

34. Tang X, Qin H, Gu X, Fu X. China's landscape in regenerative medicine. Biomaterials. 2017;124:78-94.

35. Kaku M, Akiba Y, Akiyama K, Akita D, Nishimura M. Cell-based bone regeneration for alveolar ridge augmentation — cell source, endogenous cell recruitment and immunomodulatory function. J Prosthodont Res. 2015;59(2):96-112.

36. Yakovlev S, Medved L. Effect of fibrinogen, fibrin, and fibrin degradation products on transendothelial migration of leukocytes. Thromb Res. 2018; 162:93-100.

37. Gligorijević N, Zámorová Križáková M, Penezić A, Katrlík J, Nedić O. Structural and functional changes of fibrinogen due to aging. Int J Biol Macromol. 2018:108:1028-34.

38. Kim S, Kawai T, Wang D, Yang Y. Engineering a dual-layer chitosan-lactide hydrogel to create endothelial cell aggregate-induced microvascular networks in vitro and increase blood perfusion in vivo. ACS Appl Mater Interfaces. 2016;8(30):19245-55.

39. Mishra R, Roux BM, Posukonis M, Bodamer E, Brey EM, Fisher JP, Dean D. Effect of prevascularization on in vivo vascularization of poly (propylene fumarate)/fibrin scaffolds. Biomaterials. 2016;77:255-66.

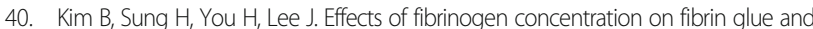
bone powder scaffolds in bone regeneration. J Biosci Bioeng. 2014;118(4):469-75.

41. Nacopoulos C, Dontas I, Lelovas P, Galanos A, Vesalas A, Raptou P, Mastoris $\mathrm{M}$, Chronopoulos E, Papaioannou N. Enhancement of bone regeneration with the combination of platelet-rich fibrin and synthetic graft. J Craniofac Surg. 2014;25:2164-8.

42. Horimizu M, Kubota T, Kawase T, Nagata M, Kobayashi M, Okuda K, Nakata $\mathrm{K}$, Yoshie H. Synergistic effects of the combined use of human-cultured periosteal sheets and platelet-rich fibrin on bone regeneration: an animal study. Clin Exp Dent Res. 2017;3(4):134-41.

43. Wang LH, Tsai HC, Cheng YC, Lin CY, Huang YL, Tsai CH, Xu GH, Wang SW, Fong YC, Tang CH. CTGF promotes osteosarcoma angiogenesis by regulating miR-543/angiopoietin 2 signaling. Cancer Lett. 2017;391:28-37.

44. Shi-Wen X, Leask A, Abraham D. Regulation and function of connective tissue growth factor/CCN2 in tissue repair, scarring and fibrosis. Cytokine Growth Factor Rev. 2008;19(2):133-44.

45. Liu S, Chuang S, Hsu C, Tsai C, Wang S, Tang C. CTGF increases vascular endothelial growth factor-dependent angiogenesis in human synovial fibroblasts by increasing miR-210 expression. Cell Death Dis. 2014;5(10): e1485.

46. Grote K, Salguero G, Ballmaier M, Dangers M, Drexler H, Schieffer B. The angiogenic factor CCN1 promotes adhesion and migration of circulating CD34+ progenitor cells: potential role in angiogenesis and endothelial regeneration. Blood. 2007;110(3):877-85.

47. Wang X, Lemaire SA, Chen L, Shen YH, Gan Y. Increased collagen deposition and elevated expression of connective tissue growth factor in human thoracic aortic dissection. Circulation. 2006:114(1 Suppl):I200-5.

48. Xu K, Sun Y, Kh Al-Ani M, Wang C, Sha Y, Sung KP, Dong N, Qiu X, Yang L. Synergistic promoting effects of bone morphogenetic protein 12/ connective tissue growth factor on functional differentiation of tendon derived stem cells and patellar tendon window defect regeneration. Biomech. 2018;66:95-102. 
49. Jia P, Hu Y, Li G, Sun Y, Zhao J, Fu J, Lu C, Liu B. Roles of the ERK1/2 and PI3K/PKB signaling pathways in regulating the expression of extracellular matrix genes in rat pulmonary artery smooth muscle cells. Acta Cir Bras. 2017;32(5):350-8.

50. Ferreira $C L$, Abreu FAMD, Silva GAB, Silveira FF, Barreto LBA, Paulino TDP, Miziara MN, Alves JB. TGF- $\beta 1$ and BMP-4 carried by liposomes enhance the healing process in alveolar bone. Arch Oral Biol. 2013;58(6):646-56.

51. Mu H, Wang L. Effect of therapeutic ultrasound on brain angiogenesis following intracerebral hemorrhage in rats. Microvasc Res. 2015;102:11-8.

52. Helling ER, Dev VR, Garza J, Barone C, Nelluri P, Wang PT. Low fistula rate in palatal clefts closed with the Furlow technique using decellularized dermis. Plast Reconstr Surg. 2006;117(7):2361-5

53. Gonzaga VF, Wenceslau CV, Lisboa GS, Frare EO, Kerkis I. Mesenchymal stem cell benefits observed in bone marrow failure and acquired aplastic anemia. Stem Cells Int. 2017:2017:1-12.

54. Wu J, Wang Q, Fu X, Wu X, Gu C, Bi J, Xie F, Kang N, Liu X, Yan L, et al. Influence of immunogenicity of allogeneic bone marrow mesenchymal stem cells on bone tissue engineering. Cell Transplant. 2016;25(2):229-42.

55. Ikeguchi R, Kakinoki R, Ohta S, Oda H, Yurie H, Kaizawa Y, Mitsui H, Aoyama T, Toguchida J, Matsuda S. Recipient bone marrow-derived stromal cells prolong graft survival in a rat hind limb allotransplantation model. Microsurgery. 2017;37(6):632-40.

Ready to submit your research? Choose BMC and benefit from:

- fast, convenient online submission

- thorough peer review by experienced researchers in your field

- rapid publication on acceptance

- support for research data, including large and complex data types

- gold Open Access which fosters wider collaboration and increased citations

- maximum visibility for your research: over $100 \mathrm{M}$ website views per year

At BMC, research is always in progress.

Learn more biomedcentral.com/submissions 